\title{
Unidirectional direct current in coupled nanomechanical resonators by tunable symmetry breaking
}

\author{
M. Prada, ${ }^{1,2,{ }^{*}}$ G. Platero, ${ }^{1}$ and D. Pfannkuche ${ }^{2}$ \\ ${ }^{1}$ Instituto de Ciencias Materiales de Madrid, ICMM-CSIC, Sor Juana Ines de la Cruz 3, Madrid, Spain \\ ${ }^{2}$ I. Institut für Theoretische Physik, Universität Hamburg, Jungiusstrasse 9, 20355 Hamburg, Germany \\ (Received 27 June 2013; revised manuscript received 19 November 2013; published 27 January 2014)
}

\begin{abstract}
We investigate theoretically the nonlinear dynamics of the most fundamental component of a bistable coupled oscillator. Under a weak radio frequency excitation, the resonator is parametrically tuned into self-sustained oscillatory regimes. The transfer of electrons from one contact to the other is then mechanically assisted, generating a rectified current. The direction of the rectified current is, in general, determined by the phase shift between the mechanical oscillations and the signal. However, we locate intriguing parametrical regions of unidirectional rectified current, resulting from spontaneous parity-symmetry breaking. In these regions, a dynamical symmetry breaking is induced by the nonlinear coupling of the mechanical and electrical degrees of freedom. The achieved unstable regime favors then a unidirectional response, resulting in a direct, positive electric current. When operating within the Coulomb blockade limit, the charge balance in the oscillators perturbs drastically the mechanical motion, causing large accelerations that further enhance the shuttle response. Our results suggest a practical scheme for the realization of a self-powered device in the nanoscale.
\end{abstract}

DOI: 10.1103/PhysRevB.89.045426

PACS number(s): 85.85.+j, 05.45.Xt, 73.23.Hk

Bifurcations are closely linked to catastrophes in systems operating in a nonlinear regime. In general terms, a system experiences a catastrophe when a smooth change in the value of a parameter results in a sudden change in the response of the system. The response is then termed as parametrically driven. Interest in parametrically driven nonlinear dynamics of nanoelectromechanical systems (NEMS) has grown rapidly over the last few years [1-3]. NEMS offer the possibility to realize nanomechanical switches [4], circuits [5-9], electronic transducers $[10,11]$, current rectifiers $[12,13]$, or high-sensitive charge [14], spin [15-17], and mass sensors [18-20], as well as the general study of nonlinear dynamics of oscillators and resonators [21-26]. In these systems charge transport is mechanically assisted and typically driven by an rf excitation of tunable intensity and frequency. When the mechanical degrees of freedom couple to the rf excitation, the system may enter a regime of strong nonlinear response as the frequency and the intensity of the excitation are slowly varied, hence, parametrically driven. Understanding behavior of these devices in the nonlinear regime can thus point to strategies for engineering self-powered NEMS-based devices [27].

Back in 1998 Gorelik et al. [28] proposed a nanomechanical transistor based on a vibrating shuttle between two contacts. The mechanical motion of the shuttle perturbs the charge balance, causing accelerations that could further enhance the response of the shuttle. Scheible et al. [14] experimentally realized such an electron shuttle in the form of a single nanopillar vibrating in a flexural mode, and observed a frequency dependent ratchet behavior. Following these experimental results, Pistolesi et al. [13] showed theoretically that the single electron shuttle can indeed act as a rectifier. More recently, Kim et al. [29-31] realized a nanomechanical shuttle capable of transferring electrons mechanically at room temperature in

\footnotetext{
*mprada@physnet.uni-hamburg.de
}

the Coulomb blockade regime. Their prototype consisted of two coupled Si nanopillars with a metallic island on top, as depicted in Fig. 1(a). The nanopillars are placed between two electrodes operating at high frequencies $(\sim 0.1 \mathrm{GHz})$. One of the great advantages of this system is that co-tunneling events are dramatically suppressed in a flexural oscillatory mode where the center of mass is at rest [see Fig. 1(a)]. Another is the obtention of a rectified dc signal resulting from spontaneous symmetry breaking [12,29].

In this paper we investigate the dynamics of the most fundamental component for the realization of bistable oscillators: a double coupled shuttle NEMS. In previous work by Ahn et al. [12], the symmetry on the phase portraits of the solutions prevented a preferred direction for the current. However, we report here on spontaneous parity-symmetry breaking due to the nonlinear coupling of the mechanical and electrical degrees of freedom. The parity-symmetry breaking results in the obtention of unidirectional direct currents. We demonstrate that parametrically controlled current flow and largely amplified response excel the double shuttle as an optimal device. We explore the parametric excitations within the Coulomb blockade limit, where abrupt, almost instantaneous increments and decrements of the charge difference in the nano-islands cause the average electrostatic force to change periodically in a square-wave fashion. Corresponding electromechanical instabilities open access to regimes of efficient charge pumping.

We start with a purely classical description of the circuit depicted in Fig. 1(b), as commonly found in literature $[12,13,28]$. The mechanical degree of freedom (see Fig. 1) is described in terms of the relative displacement of two islands $x=x_{1}-x_{2}$. The dynamics within the quasiadiabatic limit $[12,32]$ is governed by the force exerted by an average electric field $-V(t) / L$ on the coupled oscillators, yielding

$$
\ddot{x}+\frac{\gamma}{\omega_{0}} \dot{x}+x=\frac{e V(t)}{k L} n(t),
$$


(a)

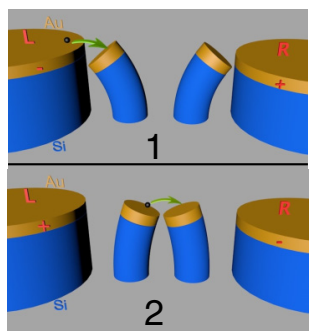

(b)

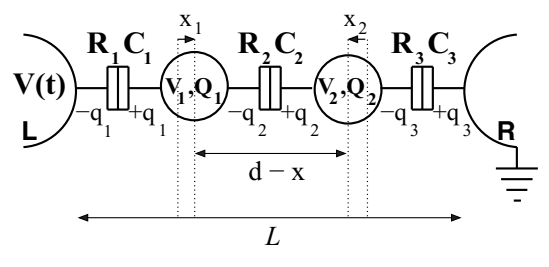

FIG. 1. (Color online) (a) Sketch showing mechanically assisted electronic transport in Si nanopillars with a metallic island on top. The black dot represents the transferred electron. The flexural mode which originates an efficient dc current has the center of mass at rest. (b) Circuit representation: Two metallic islands are capacitively coupled to each other and to both electrodes, $\mathrm{L}$ and $\mathrm{R}$.

where $n(t)=-\left(Q_{1}-Q_{2}\right) / e, Q_{1,2}$ denotes the charge in the nano-islands, with $Q_{i}=q_{i}-q_{i+1}, q_{i}$ being the charge in the $i$ th junction [see Fig. 1(b)], $k=m \omega_{0}^{2}$, with $m$ being the mass of a pillar and $\omega_{0}$ is the oscillator eigenfrequency, $\gamma$ denotes the dynamic damping, and $L$ is the distance between the electrodes. When the flexural modes of the nanopillars are excited, the resistances and mutual capacitances of each junction become sensitive to the displacements: $C_{i} \simeq C_{i}^{0} /\left(1+x_{i}\right)$ and $R_{i}=R_{i}^{0} \exp \left(x_{i} / \lambda\right)$, where $\lambda$ is the phenomenologically introduced tunneling length. We take $R_{1}^{0}=R_{3}^{0}=R_{2}^{0} / 2=R$ and $C_{1}^{0}=C_{3}^{0}=2 C_{2}^{0}=C$, consistent with previous results [30]. Classical circuit analysis for the doubly charged shuttle gives $V(t)=\sum_{i} q_{i} / C_{i}, i=1,3, q_{i} / R_{i} C_{i}=q_{j} / R_{j} C_{j}, j \neq i$, allowing us to express the charge on each island in terms of the relative displacement $x$ [33],

$$
Q_{1}=-Q_{2} \simeq \frac{C V(t)}{2}\left[\tanh \frac{3 x}{4}-\frac{\lambda}{d} x e^{\frac{3 x}{2}}\right],
$$

with $d$ being the distance at rest between the shuttles. For small oscillations, $x \lesssim 1$, we expand Eq. (2) to third order. Equation (1) becomes a modified Mathieu equation,

$$
\ddot{x}+x+\frac{\gamma}{\omega_{0}} \dot{x}+\alpha(\sin \omega t+\beta)^{2}\left[x-\frac{2 \lambda}{d} x^{2}-\frac{3}{16} x^{3}\right]=0 .
$$

The dimensionless parameter $\alpha=3 C V_{0}^{2} / 4 m L \lambda \omega_{0}^{2}$ quantifies the strength of the rf excitation, being the ratio of the electric $\left(\sim C V_{0}^{2} / L\right)$ and mechanical forces $\left(\sim m \lambda \omega_{0}^{2}=k \lambda\right)$, with $k$ being the spring constant. $V_{0}$ and $\beta$ relate to the applied voltage $V(t)=V_{0}(\sin \omega t+\beta)$. Throughout this work we take typical experimental values for $\lambda / d=0.1$ and $\gamma / \omega_{0}=10^{-2}$. Within the weak electromechanical coupling limit we parametrize the damping and the excitation strength with an arbitrarily small $\varepsilon, \gamma \sim \varepsilon \gamma_{1}$ and $\alpha \sim \varepsilon \alpha_{1}$. We consider the resonant modes of the system, $\omega \simeq \omega_{0}\left(p+\varepsilon \delta_{\omega}\right)$, where $\delta_{\omega}$ indicates the deviation from the natural resonance and $p$ is the winding number $[28,29]$. This defines two time scales, the "stretched" time $z=\omega t$, and the "slow" time $\eta=\varepsilon t$. We seek for steady oscillatory solutions $x(\eta, z)=A(\eta) \cos z / p-$ $B(\eta) \sin z / p$. Note that the mechanical oscillations can be alternatively expressed as $x(\eta)=r_{0}(\eta) \cos [\omega t-\varphi(\eta)]$, with $r_{0}^{2}=A^{2}+B^{2}$, and $\varphi=\arctan B / A$ defining the phase
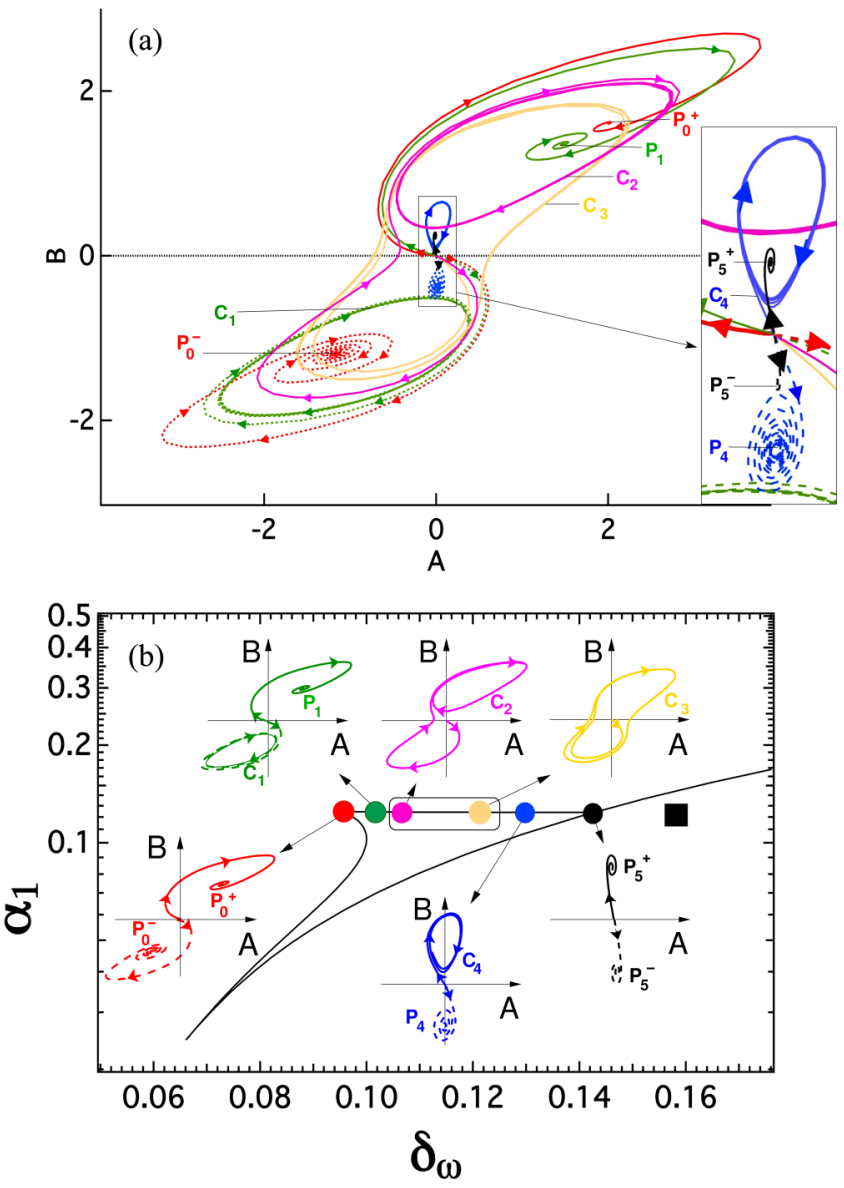

FIG. 2. (Color online) (a) Phase portraits for the fundamental mode $p=1$. Inset: Magnification around the origin. (b) Corresponding bifurcation diagram in parameter space. The rectangle marks a band of unidirectional current. Insets: Phase portraits where symmetry breaking occurs.

between the mechanical oscillations and the voltage. Following the Poincaré-Lindstedt method, we find linearized differential equations for the coefficients $A$ and $B$ [33]. Phase portraits and bifurcation diagrams are finally computed using evaluation routines [34].

The phase portraits in the first unstable region are shown in Fig. 2(a), with the corresponding bifurcation diagram in Fig. 2(b). We chose six representing points along the parametric region of multiple stability. The associated phase portraits for each point are depicted in the insets [from Fig. 2(a)]. For these, the initial conditions were set near equilibrium, $B_{0}=b_{0}$ $\left(B_{0}=-b_{0}\right)$ for the solid (broken) traces, with arbitrarily small $b_{0}$. The stable solutions are either attractor points $P_{i}^{ \pm}$ or cycles $C_{i}, i=0, \ldots, 4$. This results in electromechanical instabilities, in the sense that even if the pillars are initially nearly at rest, the electrostatic field will cause them to oscillate.

A pitchfork bifurcation is observed as we move from the left in the unstable region. The origin undergoes a transition, from a stable spiral to a saddle, where two nontrivial quasisymmetric attractors appear (red traces, $P_{0}^{ \pm}$). A supercritical AndronovHopf bifurcation causes one of the attractors to become an asymptotic curve $\left(C_{1}\right)$. Under further parametric variations 

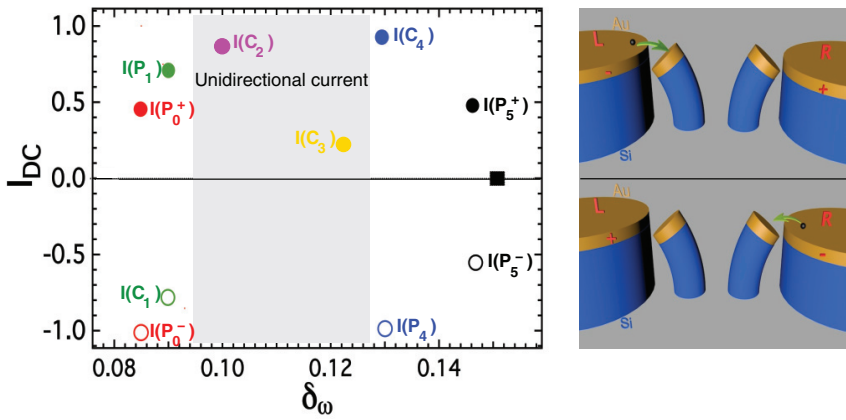

FIG. 3. (Color online) Computed $I_{\mathrm{dc}}$ at the stable solutions (arbitrary units). The solid (open) dots are associated to the solid (broken) trajectories of the insets of Fig. 2(b) with the corresponding color code. In the shaded region the direct current occurs only left to right. Right insets: Sketch of the movement of the pillars. For $B>0$ $(<0)$, the mechanical oscillation is in (out of) phase with the applied rf signal (see sign on the contacts), and hence, $I_{\mathrm{dc}}$ results positive (negative).

a homoclinic bifurcation results in the birth of an unique limit cycle $\left(C_{2}, C_{3}\right)$. The asymmetry of the cycles results in an unique unidirectional current (see below). The rectangle marks the parametric region where unidirectional current is obtained.

The time-average direct current is then obtained for each asymptotic solution $\left(P_{i}, C_{i}\right)$ by integrating over a period the current across a junction,

$$
I_{\mathrm{dc}}=\frac{\omega}{4 \pi R} \int_{t_{0}}^{t_{0}+T} d t \frac{V(t) e^{x}}{1+e^{3 x / 2} \cosh \left(\frac{X}{2}\right)} .
$$

Here $X$ is the center of mass displacement, which in most of the cases remains at rest [12]. The results are summarized in Fig. 3. An intuitive picture is sketched in the right insets of Fig. 3: For $B>0$, the mechanical oscillations are always in phase with the voltage and electrons are flowing from left to right. However, for $B<0$, the oscillators are out of phase, resulting in a negative rectified current. Outside the unstable region [black square of Fig. 2(b)] the trivial solution $(A=B=0)$ is an attractor, and no direct current is observed. The sign of the current in typical bistable regions is then determined by the initial conditions $\pm b_{0}$. This occurs in the red, green, blue, and black traces of Fig. 2(a), where the sign of the phase is preserved, or, in other words, the trajectory remains in one semiplane $(B>0$ or $B<0)$. The corresponding stroboscopic plot is nearly symmetric in these bistable regions, so a positive or negative current can occur. On the contrary, for the magenta and yellow traces of Fig. 2(a), only one stable asymptotic orbit is found $\left(C_{2}\right.$ and $\left.C_{3}\right)$. The lack of symmetry of the stable phase portrait result in a positive (left to right) net current independently of the initial conditions. This occurs throughout the shaded region of Fig. 3. We note that the term in $x^{2}$ of Eq. (3) breaks the parity symmetry of the system: For a given solution $x(t),-x(t)$ is no longer a solution. This term results from relative variation of the individual capacitances and resistances with the positions of the islands. Hence, we conclude that a dynamical symmetry breaking occurs due to the nonlinear coupling of the mechanical and electrical degrees of freedom. We stress that the sign

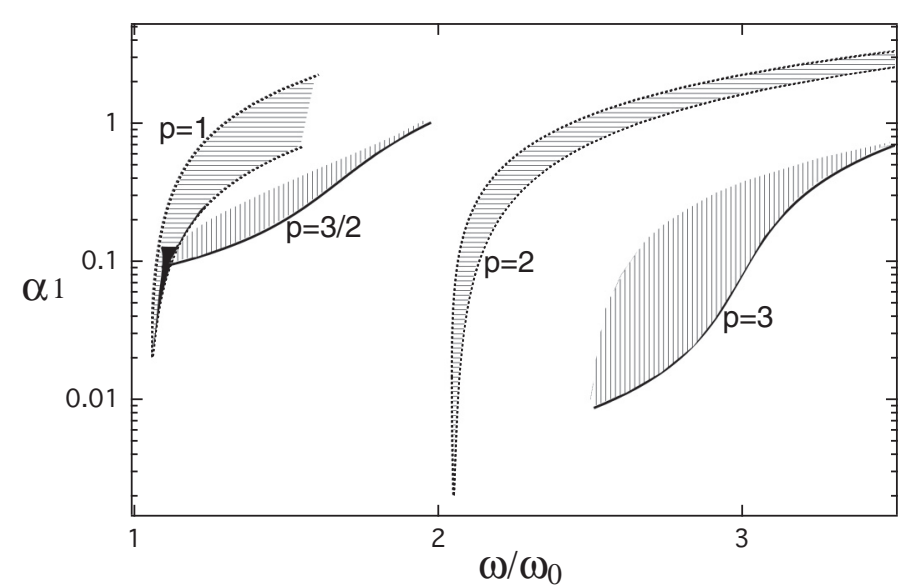

FIG. 4. Bifurcation diagram showing regions with multiple stability in the areas where the origin is unstable (horizontally hatched, $p=1,2$ ) or stable (vertically hatched, $p=3 / 2,3$ ). The dark region in $p=1$ corresponds to Fig. 2(b).

of the current is then parametrically controlled, suggesting an energy harvester for self-powered nanosystems [35], or a nanobattery.

We now study the response on the relevant higher excitation frequency regions, i.e., $p=3 / 2,2,3$ in Eq. (4). For these, nontrivial solutions are found in the hatched areas indicated in Fig. 4. The case $p=2$ is of particular interest, as there exists a region in parameter space for which the origin becomes unstable. We then have a situation similar to the one of Fig. 2, within a different region of parameter space (see Fig. 3). For the cases $p=3 / 2,3$, the origin of the phase plane is always an attractor, and hence, solutions with initial conditions in its proximity will vanish. However, another three attractors exist around a small area of the phase plane. Only when the initial conditions are close to these attractors, self-sustained oscillations are possible for this mode, but no electromechanical instabilities are found.

Next, we evaluate the dynamics of the islands within the Coulomb blockade limit, for which $C V \simeq e$. In such a picture, abrupt increments and decrements of charge in the metallic islands occur almost instantaneously, separated by periodic time intervals [36]. We use the master equation in terms of the excess electrons in the islands $n_{1,2}$,

$$
\dot{P}_{n_{1}, n_{2}}=\sum_{n_{1}, n_{2} ; k} \Gamma_{n_{1}^{\prime}, n_{2}^{\prime} \rightarrow n_{1}, n_{2}}^{k} P_{n_{1}^{\prime}, n_{2}^{\prime}}-\Gamma_{n_{1}, n_{2} \rightarrow n_{1}^{\prime}, n_{2}^{\prime}}^{k} P_{n_{1}, n_{2}}
$$

where the tunneling rate at the $k$ th junction is given, according to the orthodox theory, by $\Gamma_{i \rightarrow j}^{k}=\mu_{i j}(t) / e^{2} R_{k}(t)$ $\left[1-e^{-\mu_{i j}(t) / k_{B} T}\right]$, with $\mu_{i j}$ being the decrease of free energy when the tunneling event occurs. We solve Eq. (5) by direct integration and get the occupation on each island $\left\langle n_{i}(t)\right\rangle$. Figure 5(a) shows the steady state solution for $\langle n(t)\rangle$. As it can be seen, charge transfer occurs in the points of maximal deflection. The relative charge is nearly a square wave, $\langle n(t)\rangle \sim n_{\mathrm{av}}+4 n_{0}\left(\cos \omega_{0} t-\cos 3 \omega_{0} t\right) / \pi$. Inserting this expression into Eq. (1), we find, as before, linearized equations for the coefficients $A$ and $B$ of the oscillatory 

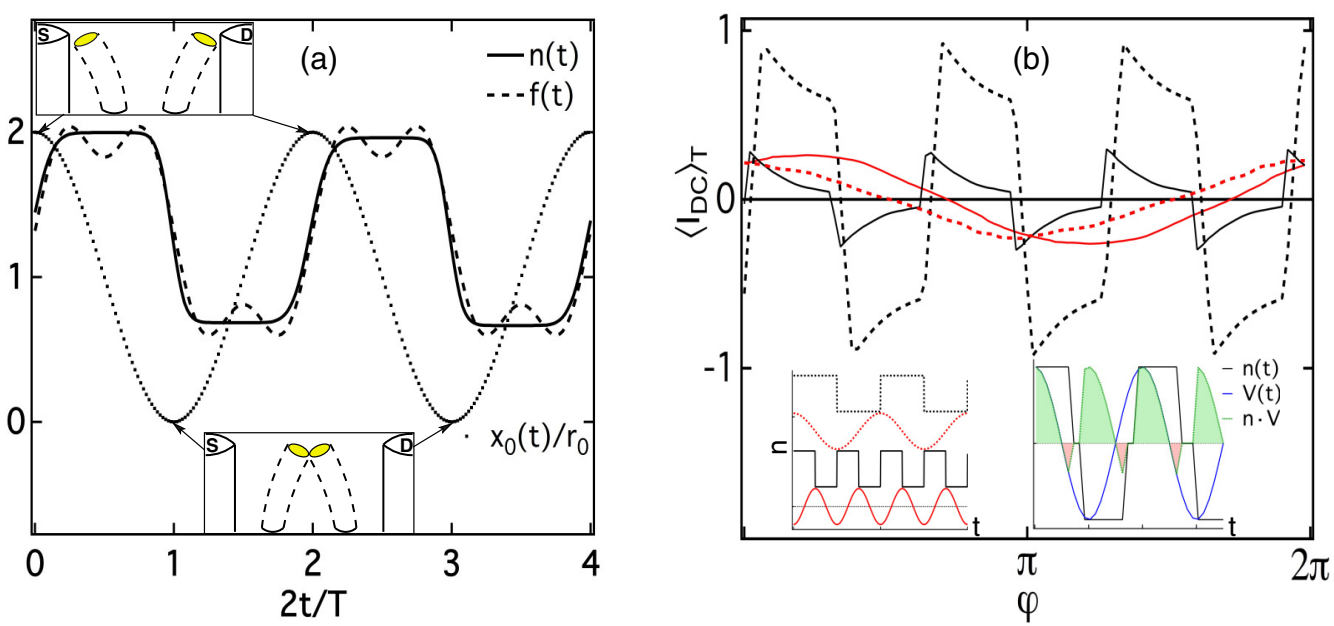

FIG. 5. (Color online) (a) $n(t)$ calculated using the master equation (solid curve), square-wave fit $f(t)=n_{\mathrm{av}}+4 n_{0}\left(\cos \omega_{0} t-\cos 3 \omega_{0} t\right) / \pi$ (broken curve), and normalized relative displacement $x_{0}(t) / r_{0}$ (dotted curve). The insets mark the points of maximal displacement for the pillars. (b) Averaged dc current per period for four different charge oscillations. Lower-left inset: The corresponding charge oscillations. Lower-right inset: $n(t)$ (solid black curve) and rf signal (blue trace). The resulting direct current per period is then proportional to the green shaded areas minus the pink ones.

solutions,

$$
\begin{aligned}
& 2 \frac{d A}{d \eta}=-A \gamma_{1}-\frac{2 \delta_{\omega}}{p} B+\alpha_{1}^{\prime} n_{\mathrm{av}} \delta_{p, 1}-\frac{\alpha_{1}^{\prime}}{6}\left(2 \delta_{p, 2}+\delta_{p, 4}\right), \\
& 2 \frac{d B}{d \eta}=-B \gamma_{1}+\frac{2 \delta_{\omega}}{p} A+\alpha^{\prime} \beta n_{0},
\end{aligned}
$$

with $\alpha^{\prime}=e V_{0} / L k \lambda$ and $\alpha_{1}^{\prime}=\varepsilon \alpha^{\prime}$. It is straightforward to see that Eq. (6) has one nontrivial attractor in the phase plane: In the absence of $V_{\mathrm{dc}}(\beta=0)$, stable points are found only around subharmonics with $p=1,2$, or $4,\left(A_{p}, B_{p}\right)=$ $a_{p}\left(\gamma, \gamma_{p}\right)$, with $\gamma_{p}=2 \delta_{\omega} / p, a_{1}=\alpha^{\prime} n_{\mathrm{av}} /\left(\gamma^{2}+\gamma_{1}^{2}\right), \quad a_{2}=$ $-\alpha^{\prime} n_{0} / 3\left(\gamma^{2}+\gamma_{2}^{2}\right)$, and $a_{4}=-\alpha^{\prime} n_{0} / 6\left(\gamma^{2}+\gamma_{4}^{2}\right)$. In contrast, under a finite dc bias, the left-right symmetry is broken, and oscillatory solutions are found for any frequency at $\left(A_{p}, B_{p}\right)=\alpha \beta n_{0}\left(\gamma_{p},-\gamma\right) /\left(\gamma^{2}+\gamma_{p}^{2}\right)$. Let us focus now on the existence of a self-sustained oscillation. In an oscillatory mode $x \sim r_{0} \cos \left(\omega_{0} t+\varphi\right)$, the absorbed power by the oscillator per unit cycle is obtained by averaging over a period the last term of Eq. (1):

$$
\left\langle W_{\mathrm{a}}\right\rangle \simeq\left\langle\alpha^{\prime} n(t) \dot{x}(t)(\sin \omega t+\beta)\right\rangle,
$$

whereas the dissipated power is given by the damping term $\left\langle W_{\text {dis }}\right\rangle=\left\langle\dot{x}^{2}\right\rangle \gamma / \omega_{0}$. If this amount is larger than the dissipated power, $\left\langle W_{\mathrm{a}}\right\rangle \gtrsim\left\langle W_{\text {dis }}\right\rangle$, self-sustained oscillations [37] are expected. This occurs when $\max \left\{\alpha^{\prime} n_{\mathrm{av}, 0}, \alpha^{\prime} n_{0} \beta\right\} \gtrsim r_{0} \gamma / \omega_{0}$, with the appropriate phase $\varphi$. The amplitude of the oscillations could then be large enough to reach the Fowler-Nordheim tunneling limit [38], with a subsequent enhancement of the direct current due to field emission. Hence, under a finite dc bias, bands of instabilities occur for a particular value of the applied rf power $\alpha$. Recent experimental data confirm the existence of such bands [31].

To illustrate the importance of the phase between the mechanical oscillations and the rf signal, we compute the current for a few fictitious charge oscillations $n(t)$. Figure 5(b) shows the average direct current (number of transferred electrons per cycle) as a function of the phase $\varphi=\arctan \{B / A\}$ for four different charge oscillations, sketched in the lower-left inset. The function has the same period as the signal (solid traces) or twice (broken traces), and is a square wave (black) or a sinusoidal (red). It is evident from the figure that the square-wave function with a periodicity twice the signal one (broken black traces) shows the most effective current on a broad relative phase range. A square-wave fashion variation is indeed known to pump energy more effectively than a sinusoidal variation [39]. This results in a rapid and enhanced response of the system, a very feature desired for industrial applications. The current is then negative for $(2 n-1) \pi / 3 \lesssim \varphi \lesssim 2 n \pi / 3$ and positive elsewhere. We stress that the unidirectional current flow translates from the classical limit to the Coulomb blockade regime. The lower-right inset represents the rf signal (blue) and the corresponding $n(t)$ (black). The averaged current per period is then proportional to the shaded areas, which is, for this particular choice of phase $(\varphi=0)$, nonzero and positive.

In summary, we have theoretically studied a weakly ac driven coupled electron shuttle. We find multiple stability regions in parameter space with subsequent self-sustained oscillations generating a finite observable direct current. In the bistable regions, the sign of the current is bound to the relative phase of the mechanical oscillators and the signal. Subsequent dynamical symmetry breaking results in the obtention of unidirectional direct currents, a key feature in the implementation of a nanobattery. Within the Coulomb blockade regime, electromechanical instabilities are observed, which occur at any frequency in the presence of a dc bias. The tunability of the self-sustained oscillations and the unidirectionality of the current suggest a vast number of potential applications.

We are grateful to R. H. Blick and C. Kim for enlightening discussions. This work was supported by the program SB2009-0071, MAT 2011-24331, ITN Grant 234970, and GrK 1286. 
[1] L. Guillermo Villanueva, R. B. Karabalin, M. H. Matheny, E. Kenig, M. C. Cross, and M. L. Roukes, Nano Lett. 11, 5054 (2011).

[2] D. Midtvedt, Y. Tarakanov, and J. Kinaret, Nano Lett. 11, 1439 (2011).

[3] M. V. Requa and K. L. Turner, Appl. Phys. Lett. 88, 263508 (2006).

[4] A. Subramanian, A. R. Alt, L. Dong, B. E. Kratochvil, C. R. Bolognesi, and B. J. Nelson, ACS Nano 3, 2953 (2009).

[5] R. H. Blick, H. Qin, H.-S. Kim, and R. Marsland, New J. Phys. 9, 241 (2007).

[6] I. Mahboob, K. Fujiwara, A. Flurin, E. Nishiguchi, and H. Yamaguchi, Nat. Commun. 2, 198 (2011).

[7] M. D. LaHaye, J. Suh, P. M. Echternach, K. C. Schwab, and M. L. Roukes, Nature (London) 459, 960 (2009).

[8] X. Zhou, F. Hocke, A. Schliesser, A. Marx, H. Huebl, R. Gross, and T. J. Kippenberg, Nat. Phys. 9, 179 (2013).

[9] T. A. Palomaki, J. W. Harlow, J. D. Teufel, R. W. Simmonds, and K. W. Lehnert, Nature (London) 495, 210 (2013).

[10] R. He, X. L. Feng, M. L. Roukes, and P. Yang, Nano Lett. 8, 1756 (2008).

[11] S. T. Bartsch, A. Lovera, D. Grogg, and A. M. Ionescu, ACS Nano 6, 256 (2012).

[12] K.-H. Ahn, H. C. Park, J. Wiersig, and H. Jongbae, Phys. Rev. Lett. 97, 216804 (2006).

[13] F. Pistolesi and R. Fazio, Phys. Rev. Lett. 94, 036806 (2005).

[14] D. V. Scheible and R. H. Blick, Appl. Phys. Lett. 84, 4632 (2004).

[15] D. Rugar, R. Budakian, H. J. Mamin, and B. W. Chui, Nature (London) 430, 329 (2004).

[16] A. Pályi, P. R. Struck, M. Rudner, K. Flensberg, and G. Burkard, Phys. Rev. Lett. 108, 206811 (2012).

[17] L. Chotorlishvili, D. Sander, A. Sukhov, V. Dugaev, V. R. Vieira, A. Komnik, and J. Berakdar, Phys. Rev. B 88, 085201 (2013).

[18] D. Rugar and P. Grütter, Phys. Rev. Lett. 67, 699 (1991).

[19] A. K. Naik, M. S. Hanay, W. K. Hiebert, X. L. Feng, and M. L. Roukes, Nat. Nano 4, 445 (2009).

[20] V. Puller, B. Lounis, and F. Pistolesi, Phys. Rev. Lett. 110, 125501 (2013).
[21] R. Leturcq, C. Stampfer, K. Inderbitzin, L. Durrer, C. Hierold, E. Mariani, M. G. Schultz, F. von Oppen, and K. Ensslin, Nat. Phys. 5, 327 (2009).

[22] G. Weick, F. Pistolesi, E. Mariani, and F. von Oppen, Phys. Rev. B 81, 121409 (2010).

[23] A. Drews, B. Kruger, G. Selke, T. Kamionka, A. Vogel, M. Martens, U. Merkt, D. Moller, and G. Meier, Phys. Rev. B 85, 144417 (2012).

[24] G. Cohen, V. Fleurov, and K. Kikoin, Phys. Rev. B 79, 245307 (2009).

[25] T. Faust, J. Rieger, M. J. Seitner, J. P. Kotthaus, and E. M. Weig, Nat. Phys. 9, 485 (2013).

[26] T. Faust, J. Rieger, M. J. Seitner, P. Krenn, J. P. Kotthaus, and E. M. Weig, Phys. Rev. Lett. 109, 037205 (2012).

[27] Z. L. Wang and W. Wu, Angew. Chem. Int. Ed. 51, 11700 (2012).

[28] L. Y. Gorelik, A. Isacsson, M. V. Voinova, B. Kasemo, R. I. Shekhter, and M. Jonson, Phys. Rev. Lett. 80, 4526 (1998).

[29] C. Kim, J. Park, and R. H. Blick, Phys. Rev. Lett. 105, 067204 (2010).

[30] C. Kim, M. Prada, and R. H. Blick, ACS Nano 6, 651 (2012).

[31] C. Kim, M. Prada, G. Platero, and R. H. Blick, Phys. Rev. Lett. 111, 197202 (2013).

[32] A. Isacsson, L. Y. Gorelik, M. V. Voinova, B. Kasemo, R. I. Shekhter, and M. Jonson, Physica B: Condens. Matter 255, 150 (1998).

[33] M. Prada and G. Platero, Phys. Rev. B 86, 165424 (2012).

[34] MATCONT and AUTO-07p are numerical continuation software applications, freely available at http://www.matcont. UGent.be and http://indy.cs.concordia.ca/auto/

[35] J. Li, H. Yu, S. M. Wong, G. Zhang, X. Sun, P. G.-Q. Lo, and D.-L. Kwong, Appl. Phys. Lett. 95, 033102 (2009).

[36] C. Weiss and W. Zwerger, Europhys. Lett. 47, 97 (1999).

[37] H. S. Kim, H. Qin, and R. H. Blick, New J. Phys. 12, 033008 (2010).

[38] D. V. Scheible, C. Weiss, J. P. Kotthaus, and R. H. Blick, Phys. Rev. Lett. 93, 186801 (2004).

[39] E. Butikov, Comp. Sci. Eng. 3, 76 (1999). 